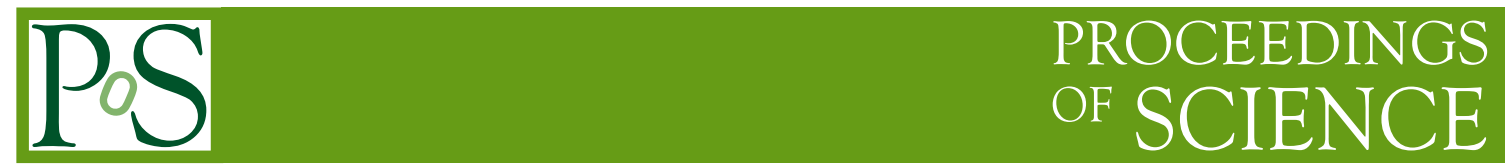

\title{
Constraining IMBH black hole masses with VLBI
}

\section{Zsolt Paragi*}

Joint Institute for VLBI in Europe

E-mail: zparagiejive.nl

\section{Michael A. Garrett}

Joint Institute for VLBI in Europe

E-mail: garrettejive.nl

\section{Andrew D. Biggs}

UK Astronomy Technology Centre

E-mail: adberoe.ac.uk

It has been suggested that some of the ultra-luminous X-ray sources may be powered by intermediate-mass black holes (IMBH). According to the recently established black hole activity plane relationship, the closest IMBHs should be detectable in the radio domain with current instrumentation. VLBI may be a powerful tool for finding the radio counterparts of ULXs and thus identifying IMBHs, because it filters out other sources such us HII regions and resolved supernova remnants. We have observed the most powerful ULX, M82 X-1, with the EVN and derive an upper limit on its mass of about $500 \mathrm{M}_{\odot}$. Future high-sensitive VLBI observations may give tighter constraints on the masses of compact objects in ULXs and may help support or rule out the IMBH hypothesis.

The 8th European VLBI Network Symposium

September 26-29, 2006

Toruń, Poland

\footnotetext{
*Speaker.
} 


\section{Ultra-luminous X-ray sources (ULXs)}

Ultra-luminous X-ray sources are off-nuclear, highly variable objects in nearby galaxies, with luminosities greatly exceeding the Eddington limit for a stellar-mass object [1.2]. Their observed luminosities indicate that they host intermediate-mass black holes (IMBH, 100-10000 solar masses) and that they represent a new type of astrophysical object [1].

There are a number of difficulties with the IMBH hypothesis. In particular, the formation of IMBHs in sufficient numbers is problematic, leading to the conclusion that the majority of ULXs are in fact stellar-mass X-ray binaries, where the X-rays from the accretion disk are geometrically beamed [3]. Other suggestions are that these sources accrete at highly super-Eddington rates [4,5.,6], or that the X-rays are coming from a relativistically beamed jet [7, 8]. However, about half-a-dozen sources show a low-energy excess in X-rays that is interpreted by some authors as thermal radiation from the accretion disk [9]. The estimated disk temperatures are much lower than expected for stellar-mass systems, indicating that they may represent the IMBH sub-class of the ULX population.

\section{Identifying intermediate-mass black holes with VLBI}

The ultimate way of identifying an accreting IMBH may be detection of synchrotron selfabsorbed radio emission from relativistic compact jets associated with these systems. In addition, radio observations provide a new way of constraining the black hole mass. To estimate the expected flux densities one may use the empirical formula of the fundamental black hole activity plane, relating the X-ray and radio luminosities to the black hole mass [10]. For the most luminous sources with $L_{\mathrm{X}} \sim 3 \times 10^{40} \mathrm{erg} \mathrm{s}^{-1}$ in nearby galaxies like M82 ( $\mathrm{D}=3-4 \mathrm{Mpc}$ ), a system hosting a black hole of $300-1000 \mathrm{M}_{\odot}$ should have a radio flux density of $\sim 50-150 \mu \mathrm{Jy}$ at cm wavelengths. The angular size of such a faint synchrotron self-absorbed radio jet with a brightness temperature of $T_{\mathrm{B}} \sim 10^{12} \mathrm{~K}$ is at the microarcsecond level and would be unresolved in VLBI observations. Because of the expected low flux densities, and the relatively poor success of detecting ULXs with connected-element interferometers, VLBI observations have never been attempted before. Recent developments in the European VLBI Network (EVN, [11]) permit sustained data rates of $1 \mathrm{Gbit} / \mathrm{s}$ to be employed using the Mark5A disk-based recording system [12]. This allows for routine detection of sources at the 50-150 $\mu \mathrm{Jy}$ level, exactly where one expects to see the more massive IMBHs. To demonstrate this, we plot the minimum detectable black hole mass in nearby galaxies with the EVN in Fig. 1.

\section{Observations of M82 X-1 with the EVN}

We observed M82 with the EVN at $1.6 \mathrm{GHz}$ on 27 Oct 2005 with the array of Cambridge, Effelsberg, 76-m Lovell Telescope (Jodrell Bank), Medicina, Noto, Onsala, 70-m Robledo and Westerbork. This well-known starburst galaxy (see PoS(8thEVN)031) hosts M82 X-1, the most extreme ULX discovered to date. This source is highly variable and the X-ray luminosity may reach $10^{41} \mathrm{erg} \mathrm{s}^{-1}$ during outbursts, indicating a mass exceeding $500 \mathrm{M}_{\odot}$ [14.15]. It is by far the best established IMBH candidate, because quasi-periodic oscillations in the X-rays provide a strong 


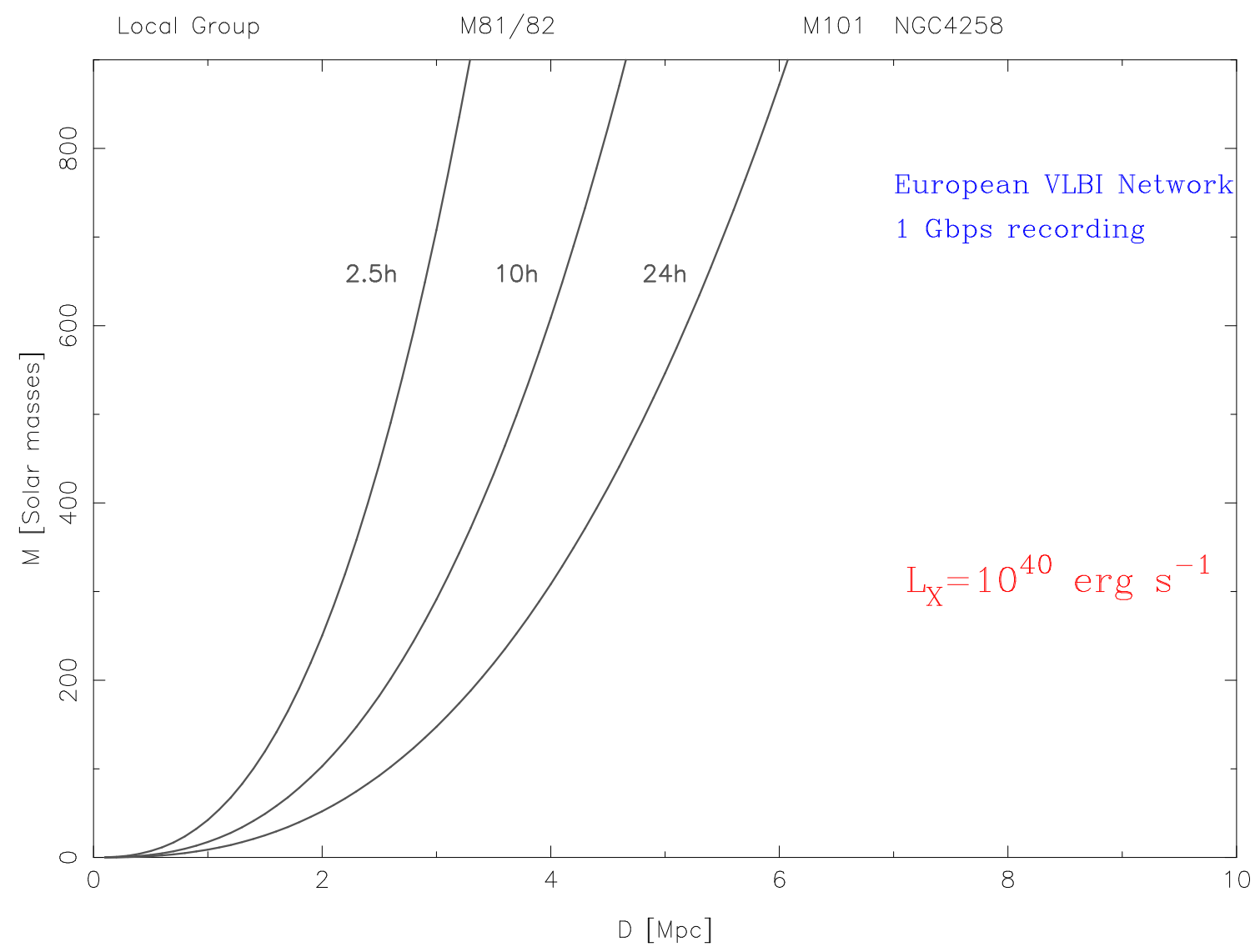

Figure 1: The minimum detectable black hole mass with the EVN in nearby galaxies. The calculations were done at $5 \mathrm{GHz}$ for sources with an X-ray luminosity of $L_{\mathrm{X}}=10^{40} \mathrm{erg} \mathrm{s}^{-1}$, using the formula by T. Maccarone [13]. The $7 \sigma$ detection limits were calculated for various on-source integration times and a recording rate of 1 Gbps.

support for the existence of an accreting massive compact object $\left(\sim 100-1000 \mathrm{M}_{\odot}\right)$ [16]. Earlier observations showed two radio sources within $\sim 1$ arcsecond around the ULX. One is a supernova observed in the mid-80s and never detected afterwards [17], while the other is the permanent mJylevel radio source M82 41.29+59.7 [18,21]. A new source, although with a low signal-to-noise ratio, was reported in recent VLA observations during X-ray flares [19]. None of these have been detected at milliarcsecond scales with VLBI before.

So far, only five compact objects have been detected with VLBI in M82 20]. We imaged these and identified three additional supernova remnant (SNR) candidates in our maps. One of them is located at the position RA $=9 \mathrm{~h} 55 \mathrm{~m} 50.0487 \mathrm{~s}, \mathrm{DEC}=+69^{\circ} 40^{\prime} 45^{\prime \prime} 942$ (J2000), well within the 2." $13 \sigma$ error box of M82 X-1 [15]. This position is in agreement with the aforementioned source M82 41.29+59.7, earlier identified as an SNR because of its steep spectral index [21]. The radio structure of $41.29+59.7$ is resolved and resembles a jet-lobe morphology, unique among other SNRs in M82. Recently, there have been two other ULXs identified with radio counterparts, likely related to jet-powered lobe emission [22,23]; our target would perfectly fit into this picture. However, there are problems with this interpretation. The resolved radio structure is in contrast with the very small angular size expected for a compact self-absorbed jet. Moreover, the observed 


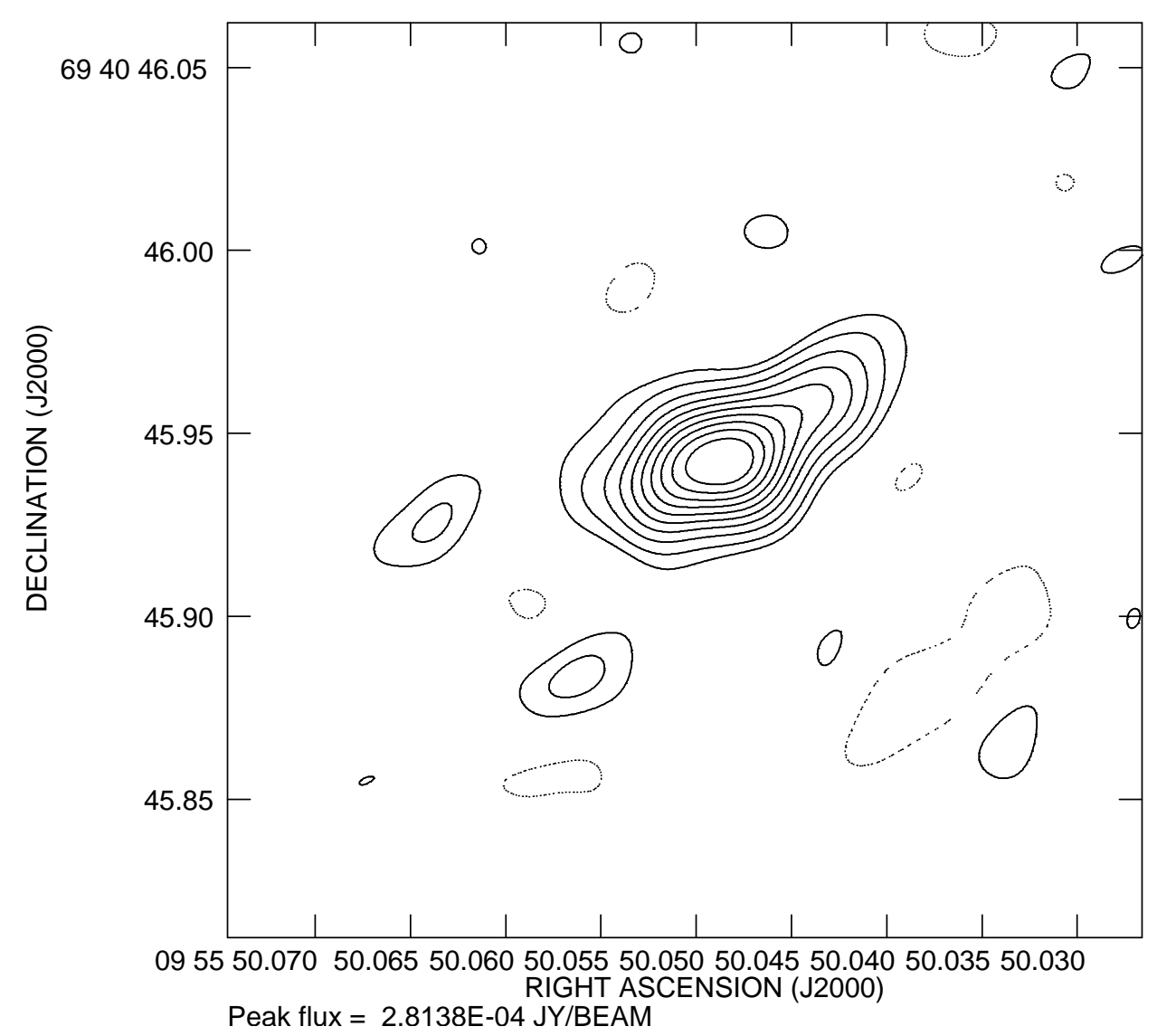

Figure 2: EVN image of M82 41.29+59.7, near the position of M82 X-1 at $1.6 \mathrm{GHz}$. The source shows a gradually resolving elongated structure, resembling a radio jet-lobe. However, deep MERLIN observations show an SNR morphology and the radio source is unlikely to be related to the ULX. The peak brightness is $281 \mu \mathrm{Jy} / \mathrm{beam}$. The contour levels are: $-0.3,0.3,0.65,1.0,1.5,2.0$, and $2.5 \times 100 \mu \mathrm{Jy} / \mathrm{beam}$. The restoring beam is $26 \times 21$ milliarcsecond with a position angle of $P A=-51$ degrees. The off-source rms noise is 15 $\mu \mathrm{Jy} / \mathrm{beam}$.

peak brightness is $281 \mu \mathrm{Jy} / \mathrm{beam}$ with a resolution of $26 \times 21$ milliarcseconds, too high for an IMBH not exceeding $1000 \mathrm{M}_{\odot}$. In an earlier VLA monitoring campaign, the source remained constant in flux density [18], there was no variation as expected for an accreting compact object. The ultimate evidence came from deep MERLIN observations that showed clear SNR morphology (T. Muxlow, priv. comm.). Our VLBI image likely shows a hotspot of the SNR.

\section{Conclusion}

We conclude that the radio counterpart of M82 X-1 remained undetected in our experiment. There were no other radio sources within the 2." 1 CHANDRA error box of M82 X-1 with a $4.5 \sigma$ upper limit of $67 \mu \mathrm{Jy} / \mathrm{beam}$. In particular, we did not detect the probable new source [19] with a $3.5 \sigma$ upper limit of $50 \mu \mathrm{Jy} / \mathrm{beam}$. At centimeter wavelengths, for the typical X-ray luminosity of $L_{\mathrm{X}}=3 \times 10^{40} \mathrm{erg} \mathrm{s}^{-1}$ and assuming $\mathrm{D}=3.9 \mathrm{kpc}$ distance for M82 [24], these place an upper limit of about $400-600 \mathrm{M}_{\odot}$ on the black hole mass of M82 X-1. This value should be taken with 
care though, because there is a significant scatter in the black hole activity plane. But as a general conclusion, we find it unlikely that M82 X-1 harbours a very massive $\left(\geq 1000 \mathrm{M}_{\odot}\right)$ black hole since that would result in a flux density likely exceeding $100 \mu \mathrm{Jy}$. A more stringent finding is that the X-rays cannot be beamed relativistically in a jet, because that would result in beaming of the radio emission as well. Thus, our data are consistent with an IMBH less massive than previously thought. However, a more likely scenario is super-Eddington accretion coupled with geometric beaming of X-rays in a stellar-mass binary system. This has been suggested for e.g. the famous Galactic source SS433 [25,26].

\section{References}

[1] E. J. M. Colbert, R. F. Mushotzky, Astrophys. J. 519, 89 (1999).

[2] K. Makishima, A. Kubota, T. Mizuno, T. Ohnishi, M. Tashiro et al., Astrophys. J. 535, 632 (2000).

[3] A. R. King, M. B. Davies, M. J. Ward, G. Fabbiano, M. Elvis, Astrophys. J. 552, L109 (2001).

[4] S. Fabrika, A. Mescheryakov, Proc. IAU Symposium 205, p. 268 (2001).

[5] M. C. Begelman, Astrophys. J. 568, L97 (2002).

[6] A. R. King, Mon. Not. R. Astron. Soc. 335, L13 (2002).

[7] C. S. Reynolds, A. J. Loan, A. C. Fabian, K. Makishima, W. N. Brandt, T. Mizuno, Mon. Not. R. Astron. Soc. 286, 349 (1997).

[8] E. Körding, H. Falcke, S. Markoff, R. P. Fender, Astronomische Gesselschaft Abstract Series 18, P176 (2001).

[9] J. M. Miller, A. C. Fabian, M. C. Miller, Astrophys. J. 614, L117 (2004).

[10] A. Merloni, S. Heinz, T. Di Matteo, Mon. Not. R. Astron. Soc. 345, 1057 (2003).

[11] The European VLBI Network is a joint facility of European, Chinese, South African and other radio astronomy institutes funded by their national research councils.

[12] http://web.haystack.edu/tech/vlbi/mark5

[13] T. J. Maccarone, Mon. Not. R. Astron. Soc. 351, 1049 (2004).

[14] A. Ptak, R. Griffiths, Astrophys. J. 517, L85 (1999).

[15] P. Kaaret, A. H. Prestwich, A. Zezas, S. S. Murray, D.-W. Kim, R. E. Kilgard, E. M. Schlegel, M. J. Ward, Mon. Not. R. Astron. Soc. 321, L29 (2001).

[16] T. Strohmayer, R. F. Mushotzky Astrophys. J. 586, L61 (2003).

[17] P. P. Kronberg, R. A. Sramek, Science 227, 28 (1985)

[18] E. Körding, E. Colbert, H. Falcke, Astron. Astrophys. 436, 427 (2005).

[19] P. Kaaret, M. G. Simet, C. C. Lang, astro-ph/0604029

[20] A. Pedlar, T. W. B. Muxlow, M. A. Garrett, P. Diamond, K. A. Wills, P. N. Wilkinson, W. Alef, Mon. Not. R. Astron. Soc. 307, 761 (1999).

[21] A. R. McDonald, T. W. B. Muxlow, K. A. Wills, A. Pedlar, R. J. Beswick, Mon. Not. R. Astron. Soc. 334, 912 (2002). 
[22] R. Soria, R. P. Fender, D. C. Hannikainen, A. M. Read, I. R. Stevens, Mon. Not. R. Astron. Soc. 368, 1527 (2006).

[23] R. Soria, Z. Kuncic, J. W. Broderick, S. D. Ryder, astro-ph/0606080

[24] S. Sakai, B. F. Madore, Astrophys. J. 526, 599 (1999).

[25] S. Fabrika, S. Karpov, P. Abolmasov, O. Sholukhova, Proc. IAU Symposium 230, p. 278 (2006).

[26] M. C. Begelman, A. R. King, J. E. Pringle, astro-ph/0604497 